\title{
Demand-Driven Dynamic Spectrum Allocation in Heterogeneous Cellular Networks
}

\author{
Li Wang ${ }^{a}$, Huiyue $\mathrm{Yi}^{\mathrm{a}}$, Honglin $\mathrm{Hu}^{\mathrm{a}}$, Jianan Liu ${ }^{\mathrm{a}}$, Bin Chen ${ }^{\mathrm{b}}$ \\ ${ }^{a}$ Shanghai Research Center for Wireless Communications, (WiCO), Shanghai, P. R. China \\ ${ }^{b}$ Huawei Technologies Co., LTd, Shenzhen, P. R. China
}

\begin{abstract}
In this paper, we address the dynamic spectrum allocation problem in next generation cellular networks under the coordinated dynamic spectrum access (CDSA) model. Firstly, considering spectrum demands of base stations, we formulate spectrum allocation under physical interference models as a nonlinear optimization problem. Then, we propose a demand-driven dynamic spectrum allocation algorithm by transforming this nonlinear optimization problem into linear programming problem. The proposed algorithm first computes initial spectrum allocation and then applies iterative enhancement. Detailed simulations show that the proposed algorithm outperforms the existing algorithms in effective spectrum utilization efficiency and in average satisfaction degree.
\end{abstract}

Index Terms: Dynamic spectrum allocation; coordinated dynamic spectrum access; physical interference model

(C) 2011 Published by MECS Publisher. Selection and/or peer review under responsibility of the Research Association of Modern Education and Computer Science

\section{Introduction}

Spectrum has long been governed by governmental regulatory authorities who allocate fixed size spectrum blocks to specific wireless services for exclusive use. Spectrum planning is usually based on the spectrum demands of busy hour. In this way, in the rest of the time the spectrum is underutilized. On the other hand, the spectrum demands for wireless services are location dependent, which means that a substantial fraction of spectrum could not be fully utilized in regions where the demands are low. Unable to adapt to the spectrum demands variations both in time and space dimensions, traditional fixed allocation could result in significant inefficiency in spectrum utilization [1]. To improve spectrum utilization, dynamic spectrum allocation (DSA) was proposed, where the allocated spectrum resource may vary in time and space.

Buddhikot et al. [1] proposed the coordinated dynamic spectrum access (CDSA) model where a centralized entity dynamically allocates the coordinated access band (CAB) to base stations or wireless access points in the

* Corresponding author.

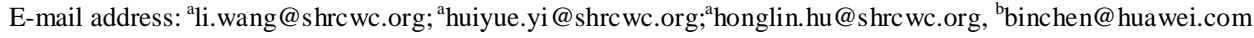


region it manages. Existing DSA algorithms fall into two categories: one is based on pairwise interference model and the other on physical interference model [2]. Under pairwise interference model, two transmissions either conflict with each other when operating on the same channel, or can use the same channel concurrently. The works in [3]-[5] consider spectrum/channel allocation under pairwise interference model, but it fails to capture the cumulated effect of interferences, which leads to large performance degradation [6]-[7]. Recently, spectrum allocation under physical interference models was investigated in [8], and an algorithm named Optimus was proposed. However, Optimus does not consider the spectrum demands from base stations. As a result, some base stations may be allocated excessive spectrum while some other base stations could not be assigned enough spectrum, which will lead to ineffective spectrum utilization efficiency. Therefore, it is necessary to consider spectrum demands of base stations in spectrum allocation algorithm to improve the spectrum utilization efficiency.

In this paper, we propose a demand-driven dynamic spectrum allocation algorithm under physical interference model by considering spectrum demands of base stations. The proposed algorithm first computes initial spectrum allocation and then applies iterative enhancement. Comparing with the existing algorithms [8], the proposed algorithm can achieve both higher effective spectrum utilization efficiency and higher average satisfaction degree.

\section{System model and problem formulation}

In this section, we first describe the system model and then formulate the problem of dynamic spectrum allocation under physical interference model.

The system model employed in this paper is CDSA model [1] which is shown in Fig.1. There is a central entity in a given region called spectrum broker who manages the allocation of a portion of spectrum to the base stations. This part of spectrum is usually referred to as $\mathrm{CAB}$. The spectrum broker divides the CAB into channels with equal sizes (e.g. $5 \mathrm{MHz}$ ). It can be assumed that the channels are homogenous and experience the same propagation conditions in average. A base station can operate on one or multiple different types of networks such as GSM/ WCDMA /LTE simultaneously. We refer to one base station with one specified network as a node. A base station operating on multiple networks is represented by multiple nodes, each of which connects to one network. Each node submits a spectrum demand to the spectrum broker in each allocation period. Based on the information of the locations of the nodes, the radio propagation model and the frequency band, it can be assumed that the spectrum broker knows the interferences between nodes. The spectrum broker grants short spectrum leases to the nodes to maximize its utility without causing interferences between nodes operating on the same channel. The spectrum lease is valid in the coverage area of a node for a given short duration.

A region controlled by spectrum broker

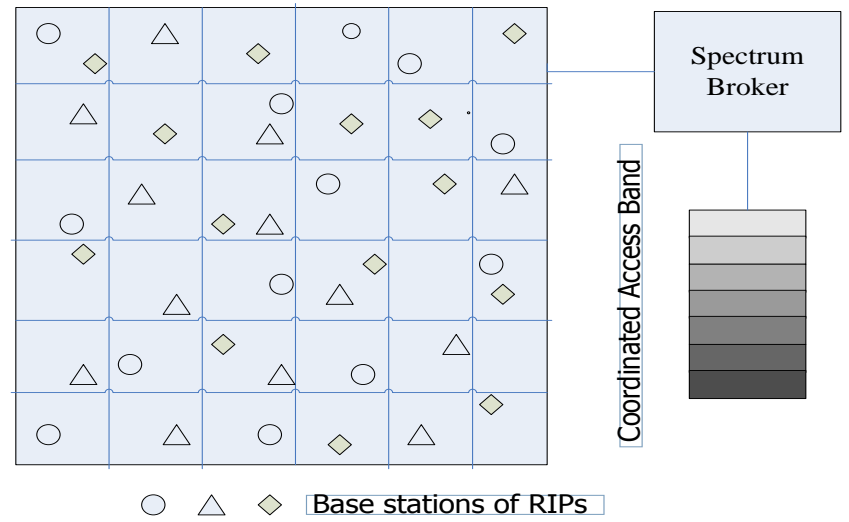

Figure 1. Coordinated dynamic spectrum access model 
We assume there are $K$ nodes in an area (e.g. a city) and a spectrum broker who manages the allocation of $M$ channels among these nodes. The objective of spectrum allocation is to find out a spectrum allocation instance which maximizes a utility function under some interference constraints.

Let $A$ denote a spectrum allocation,

$$
A=\left\{a_{m, i}\right\}, m \in[1, M], i \in[1, K]
$$

where $a_{m, i}=[0,1], a_{m, i}=1$ indicates that node $i$ is allocated to channel $m$, otherwise $a_{m, i}=0$. Thus, the number of channels allocated to node $i$ can be represented by $w_{i}=\sum_{m=1}^{M} a_{m, i}$. Then, we set $W=\left[w_{1}, w_{2}, \cdots, w_{K}\right]$.

Because pairwise interference model fails to capture the cumulated effect of interferences, we employ physical interference model to formulate the problem. In physical interference model, a successful transmission is possible if the signal to interference plus noise ratio (SINR) at the receiver is above a threshold $\beta$. Specifically, to make the transmissions on channel $m$ successful, the received SINR in the coverage area of node $i$ should satisfy:

$\operatorname{SINR}_{m, i} \geq \beta, \forall a_{m, i}=1, m \in[1, M], i \in[1, K]$

Since the SINR is location dependent, we give approximate calculation of the minimum SINR for $i$ 's coverage area as

$$
\mathrm{SINR}_{m, i}=\frac{P_{i} / r_{i}^{\alpha}}{N+\sum_{j \neq i} a_{m, j} \cdot P_{j} / d_{j, \mathrm{i}}^{\alpha}}
$$

where $P_{i}$ is the transmission power of node $i(i=1, \cdots, K), N$ is the noise power, $r_{i}$ is the communication radius of node $i, d_{j, i}$ is the smallest distance from node $j$ to node $i$ 's coverage area and $\alpha$ is the path loss exponent.

If a spectrum allocation instance $A$ satisfies the above interference constraints (2), it is considered as a valid spectrum allocation. The spectrum allocation problem is to find a valid spectrum allocation $A$ that maximizes a utility function.

\section{Demand-driven dynamic spectrum allocation algorithm}

In this section, we first define the spectrum demand model and three utility functions. Then, we give the optimal spectrum allocation solution. Finally, we describe the proposed demand driven dynamic spectrum allocation algorithm.

\section{A. $\quad$ Spectrum Demand Model and Utility Functions}

The spectrum demands of the nodes can be expressed as

$$
D=\left(d_{1}, d_{2}, \cdots, d_{K}\right)
$$

where $d_{i}$ is the number of channels required by node $i$.

There are two types of utility function. One type is revenue based utility function, and the other one is network property like spectrum utilization efficiency or network capacity. We define three network propertybased utility functions.

With respect to a valid spectrum allocation instance $A$, the normalized spectrum utilization efficiency is defined as 


$$
U_{n}(A)=U_{n}(W)=\frac{\sum_{i=1}^{K} \sum_{m=1}^{M} a_{m, i}}{M K}=\frac{\sum_{i=1}^{K} w_{i}}{M K}
$$

Considering spectrum demand of nodes, we define the effective number of channels allocated to node $i$ as $w_{i}^{\text {eff }}=\min \left(w_{i}, d_{i}\right)$. It is reasonable to do so because that the excessive channels will produce no utilization efficiency. Then, we can define the normalized effective spectrum utilization efficiency as

$$
U_{\text {eff }}(A)=U_{e f f}(W)=\frac{\sum_{i=1}^{K} w_{i}^{\mathrm{eff}}}{M \cdot K}
$$

To reflect the satisfaction degree of node $i$ for the spectrum allocation instance $A$, we define the satisfaction degree $s_{i}=\min \left(w_{i} / d_{i}, 1\right)$. Note that the satisfaction degree of node $i$ is 1 when $w_{i} \geq d_{i}$. Then, we define the average satisfaction degree as

$$
U_{\mathrm{s}}(W)=\frac{1}{K} \sum_{i=1}^{K} s_{i}
$$

Among three utility functions, the normalized effective spectrum utilization efficiency can achieve the best tradeoff between the spectrum utilization efficiency and fairness. In the following, we take the normalized effective spectrum utilization efficiency to illustrate the proposed algorithm. It is similar for the other two utility functions.

\section{B. Optimal Spectrum Allocation}

The spectrum allocation problem is to find spectrum allocation $A$ that maximizes the utility function. We formulate the optimal spectrum allocation problem as:

$$
\begin{aligned}
& \max _{A} U_{\text {eff }}(A) \\
& \text { s.t. if } a_{m, j}=1, \text { then } \operatorname{SINR}_{m, i} \geq \beta, \\
& \quad \forall m \in[1, M], i \in[1, K]
\end{aligned}
$$

This is a nonlinear optimization problem. It could only be solved by exhaustive search, which is time consuming and therefore hard for real-time implementation. Because dynamic spectrum allocation and reallocation is performed periodically with a short period (e.g., tens of minutes) [3], it is necessary to derive computationally efficient spectrum allocation algorithms. In the following, we propose an efficient demanddriven dynamic spectrum allocation algorithm.

\section{Demand-Driven Dynamic Spectrum Allocation}

The proposed algorithm consists of two steps: first compute the initial spectrum allocation, and then take the initial allocation as a clue and apply iterative enhancements to add spare channels to nodes.

\section{Step 1: Compute initial spectrum allocation}

Utilizing the idea introduced in [8] that transforms the nonlinear constraints under physical interference model into linear constraints on the number of channels allocated to the nodes, we reformulate the optimal spectrum allocation problem (8) as:

$$
\max _{W} U_{\text {eff }}(W)
$$

s.t. $\forall 1 \leq i \leq K, \quad w_{i}+\sum_{j \neq i} w_{j} \cdot I_{j, i}^{\circ} \leq M$ 
where $I_{j, i}^{\circ}=\min \left(I_{i}^{\max }, I_{j, i}\right) / I_{i}^{\max }, I_{i}^{\max }=\frac{P_{i} / r_{i}^{\alpha}}{\beta}-N$.

We can see that the utility function is nonlinear with $W=\left[w_{1}, w_{2}, \cdots, w_{K}\right]$. To make the problem resolvable through linear programming or convex optimization algorithms, we replace the original utility function $U_{\text {eff }}(A)$ defined in (6) with $U_{n}(W)$ and add a constraint that $w_{i} \leq d_{i}, \forall 1 \leq i \leq K$. Then, we rewrite the problem (9) as:

$$
\begin{aligned}
& \max _{W} U_{\mathrm{n}}(W) \\
& \text { s.t. } \forall 1 \leq i \leq K, \quad w_{i} \leq d_{i}, w_{i}+\sum_{j \neq i} w_{j} \cdot I_{j, i}^{\circ} \leq M
\end{aligned}
$$

Because the effective spectrum utilization efficiency does not increase any more when the number of channels allocated to a node reaches its demand, the optimal solution $W$ of problem (9) is achieved in the domain $w_{i} \leq d_{i}, \forall 1 \leq i \leq K$. As a result, the optimal solution of the revised problem (10) is also the optimal solution of the problem (9). Clearly, the revised problem (10) is a linear programming problem which can be worked out with efficient algorithms. Once we get the optimal solution of problem (10) using linear programming method, we abandon the decimal part and take the integral part as the approximate optimal solution. Specifically, if $\left\{w_{i}^{*}\right\}$ is the optimal solution, then the approximate optimal solution is $\left\{\left\lfloor w_{i}^{*}\right\rfloor\right\}$.

After we obtain the number of channels $\left\{\left\lfloor w_{i}^{*}\right\}\right.$ allocated to $K$ nodes, the spectrum allocation is achievable through Min-Interference Band Adjustment algorithm described in [8].

Step 2: Iterative enhancements

The linear constraints are stricter than the original physical interference constraints, and could be too conservative. We use them to produce an initial allocation, then take the initial allocation as a clue and apply heuristics based iterative enhancements to further improve spectrum utilization efficiency. The iterative enhancements algorithm work as follows:

1) Sort the nodes with descendent order of the number of channels allocated to each node in initial spectrum allocation.

2) According to the order in 1), iteratively add channels to the node until its spectrum demand has been reached or no more channels can be allocated. When adding a channel, it should not make any allocated node's SINR fall below the threshold $\beta$.

\section{Simulation results}

In this section, we present detailed performance evaluation of the proposed algorithm and compare it with Optimus. We take two utility functions defined in the previous section as performance metrics of spectrum allocation and examine the two performance metrics of these two algorithms.

\section{Simulation Setup}

We randomly place $K$ nodes within an area of $2000 m \times 2000 m$. We vary the network density by changing the number of nodes $K$ from 100 to 500. Each node can operate one of the following types of networks: WCDMA, LTE. We assume both the networks have a carrier bandwidth of $5 \mathrm{MHz}$. We consider a $\mathrm{CAB}$ with a bandwidth of $200 \mathrm{MHz}$ and we divide the $\mathrm{CAB}$ into 40 channels with equal size of $5 \mathrm{MHz}$. We assume the channel demand of each node is randomly picked from 1 to 20. Each experiment is repeated 20 times and the results are averaged over 20 experiments. Table 1 summarizes the simulation parameters. 
TABLE 1. SiMULATION PARAMETERS

\begin{tabular}{|l|c|}
\hline \multicolumn{1}{|c|}{ Parameter } & Value \\
\hline Number of channels & 40 \\
\hline Simulation area & $2000 \mathrm{~m} \times 2000 \mathrm{~m}$ \\
\hline Communication radius of node & $50 \mathrm{~m}$ \\
\hline Path loss exponent $(\alpha)$ & 2 \\
\hline TX power & $5 \mathrm{dBm}$ \\
\hline SINR threshold & $10 \mathrm{~dB}$ \\
\hline Noise power $(N)$ & $-102.5 \mathrm{dBm}$ \\
\hline Channel demand & $1 \sim 20$ \\
\hline
\end{tabular}

\section{E. Comparison to Optimus}

Fig.2 shows the normalized effective spectrum utilization efficiency comparing the proposed algorithm with Optimus. We can see the proposed algorithm has a higher normalized effective utilization efficiency than Optimus because the effective normalized spectrum utilization efficiency does not take into account the extra channels beyond the channel demand of a node. Furthermore, the iterative enhancements algorithm of the proposed algorithm can achieve higher spectrum utilization efficiency than the iterative improvements algorithm in Optimus, which will be illustrated in subsection C.

Fig.3 illustrates the average satisfaction degree comparing the proposed algorithm with Optimus. We can observe that the proposed algorithm can achieve a higher average satisfaction degree than Optimus. The reason is similar to that for the normalized effective spectrum utilization efficiency.

In addition, we observe that all the two performance metrics decrease with the network density, which is due to the increased interferences with the increased number of nodes.

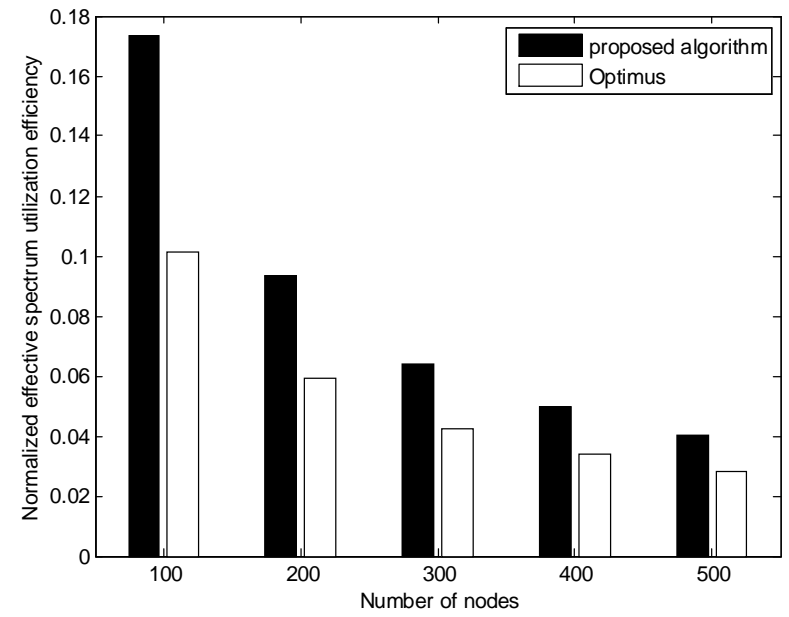

Figure 2. Normalized effective spectrum utilization efficiency comparing the proposed algorithm with Optimus 


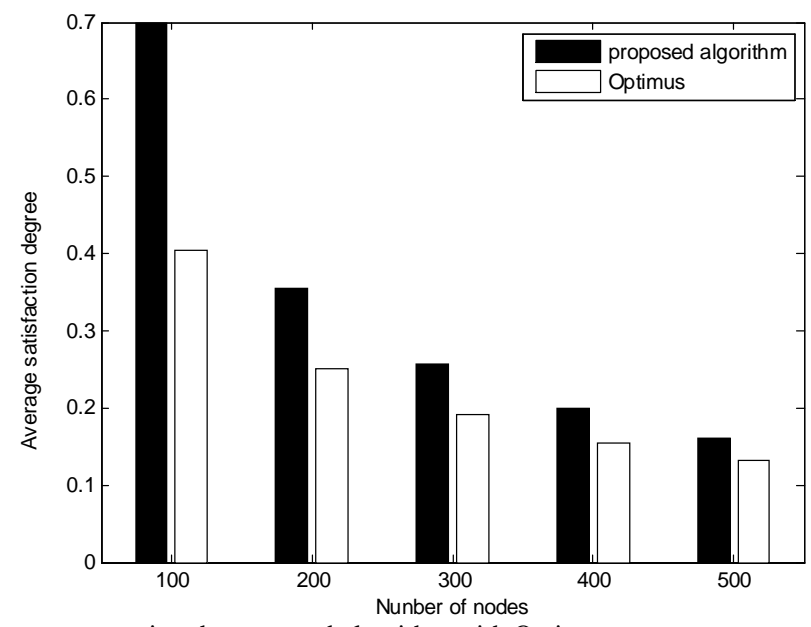

Figure 3. Average satisfaction degree comparing the proposed algorithm with Optimus

\section{F. Results for Iterative Enhancements Algorithms}

In this simulation, we assume the channel demand of each node is randomly picked from 40 to 50 . Since the total number of channels is 40 , there will be no node whose channel demand can be satisfied. Under this setting, the proposed algorithm will produce identical initial spectrum allocation result with Optimus. Thus, we can compare the iterative enhancement algorithm in the proposed algorithm with the iterative improvement algorithm in Optimus, as shown in Fig. 4. It shows that the iterative enhancement algorithm in the proposed algorithm slightly outperforms the iterative improvement algorithm in Optimus in terms of normalized spectrum utilization efficiency.

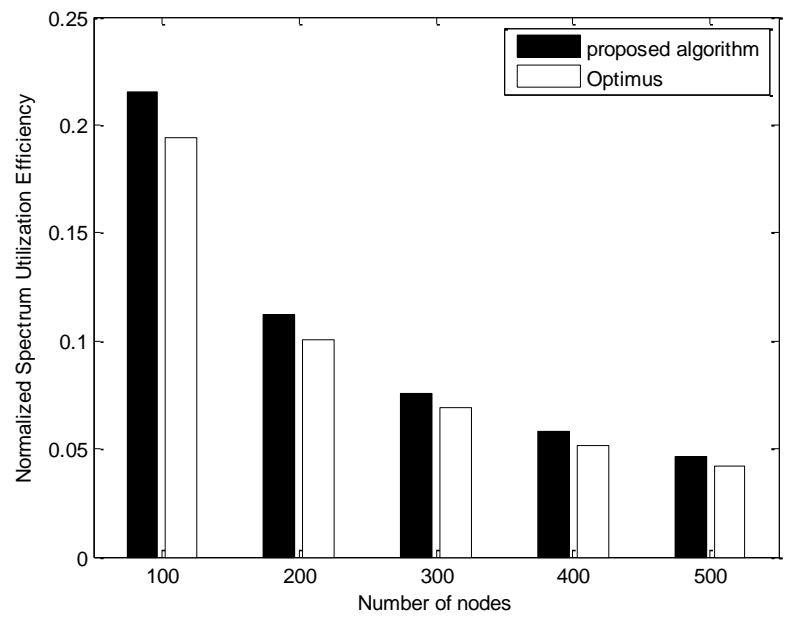

Figure 4. Normalized spectrum utilization efficiency comparing the I terative enhancement algorithm in the proposed algorithm with the iterative improvement algorithm in Optimus

\section{Conclusion and future work}

In this paper, we address the dynamic spectrum allocation problem in heterogeneous cellular networks. We have proposed an efficient demand-driven dynamic spectrum allocation algorithm, and simulations have shown that the proposed algorithm significantly outperforms the existing algorithm. Currently, the proposed algorithm can only allocate spectrum to networks with identical carrier bandwidth. In future work, we will consider the 
spectrum allocation algorithms which can deal with the spectrum allocation problem with different carrier bandwidth in future heterogeneous networks.

\section{Acknowledgements}

This work was partly supported by China natioanal key project No.2009ZX03003-002, No.2009ZX03007004, No.2011ZX03001-007-03, partly by Shanghai's Inter-Government International Cooperation Project No. 10220712000, partly by Key Project of Shanghai Science and Technology Committe No.10DJ1400302, No. 09QA1405400, No. 2010DFB10570, No.10JC1414400, No. 10ZR1429200, No. 61002017

\section{References}

[1] M. M. Buddhikot, P. Kolodzy, S. Miller, K. Ryan, and J. Evans, "DIMSUMnet: new directions in wireless networking using coordinated dynamic spectrum access," in IEEE WoWMoM, June 2005.

[2] P. Gupta, P. R. Kumar, "The capacity of wireless networks," IEEE Transactions on Information Theory, vol. 46, no. 2, pp.388-404, Mar. 2000.

[3] M. M. Buddhikot, K. Ryan, "Spectrum management in coordinated dynamic spectrum access based cellular networks," in IEEE DySPAN, November 2005.

[4] A. P. Subramanian, H. Gupta, S. R. Das, M. M. Buddhikot, "Fast spectrum allocation in coordinated dynamic spectrum access based cellular networks," in IEEE DySPAN, April 2007.

[5] L. Cao, H. Zheng, "Spectrum allocation in ad hoc networks via local bargaining," in Proc. of SECON, September 2005.

[6] G. Brar, D. Blough, P. Santi, "Computationally efficient scheduling with the physical interference model for throughput improvement in wireless mesh networks," in Proc. of ACM MobiCom, September 2006.

[7] T. Moscibroda, R. Wattenhofer, Y. Weber, "Protocol design beyond Graph-based models," in 5th Workshop on Hot Topics in Networks (HotNets). Irvine, California, USA, 2006.

[8] L. Cao, L. Yang, X. Zhou, Z.B. Zhang and H. Zheng, "Optimus: SINR-driven Spectrum Distribution via Constraint Transformation" to appear in Proc. of IEEE DySPAN ,April 2010. 